\title{
Verkündigung durch das Radio
}

\author{
von Gabriele Cakl
}

"Radio gehört gehört", so lautet der Werbespot des ORF für den Sender ,Österreich 1'.

Verkündigung versucht den christlichen Glauben, die ,Frohe Botschaft' hörbar werden zu lassen. Schon aus diesem hörbaren Grund kooperieren beide miteinander.

Anhand der Kooperation zwischen christlicher Glaubensverkündigung und dem Massenmedium Radio soll im folgenden die gegenwärtige christliche Verkündigung und ihre Medienpräsenz diskutiert werden. ${ }^{1}$

\section{Was ist Verkündigung - Radioverkündigung?}

Verkündigung ist die Vermittlung der von Gott geoffenbarten Heilstaten; in anderen Worten: das Bezeugen, Hörbar-Machen und Aktualisieren des Wortes Gottes an die Menschen.

Der Verkündigung geht das Ansprechen und die Sendung durch das Gotteswort voraus. Von menschlicher Seite aus betrachtet, bedeutet das, daß der Mensch Gottes Wort hört, Gott erfährt. Seine Antwort darauf ist der Glaube, den er als Christ nicht als individuelle Geheimlehre ansieht, sondern in der Gemeinschaft der Glaubenden, der Kirche ausspricht und austauscht.

Da die christliche Religion auf Mitteilung angelegt ist, was "grundsätzlich mit einem Öffentlichkeitsanspruch verknüpft ist" die christliche Kirche ihrem Wesen nach eine verkündende Kirche. So vielfältig sich die Gemeinschaft der Glaubenden präsentiert, so vielfältig ist auch ihre Verkündigung.

Für ihren Glauben verkündende Personen ist Verkündigung keine neutrale Nachrichtenvermittlung, sondern ein Bezeugen ihres Glau-

Mag. theol. Gabriele Cakl ist Religionspädagogin in Linz und freie Mitarbeiterin beim ORF (Abteilung Religion/Hörfunk).

1 Diesem Aufsatz liegt die Diplomarbeit der Verfasserin zugrunde: G. Cakl, Verkündigung in Alltag am Beispiel der Verkündigung durch das Radio, Salzburg 1995.

2 E. Bieger, Religiöse Rede im Fernsehen (Diss.), Salzburg 1994, 372. 
bens. ${ }^{3}$ Das Wort Gottes erfahren bedeutet, daß die Tiefe der persönlichen Existenz angesprochen und gedeutet wird und somit Sinn und Orientierung vermittelt werden können. Der angesprochene Mensch will diese Erfahrung weitersagen. Letztlich liegt es allerdings nicht in der Hand des Menschen, sondern in der Hand Gottes, wo, wann und wie das Gotteswort wirksam wird.

Verkündigung geschieht dort, wo die Intention dahintersteht. Gleichzeitig kann der Effekt oder das Ziel der Verkündigung auch unabhängig von der verkündenden Person bzw. ihrer Intention erreicht werden. Diese Unterscheidung zwischen ,bewußter' und ,unbewußter' Verkündigung ist vor allem für die Bewertung von sog. Verkündigungssendungen nicht unbedeutend.

Was mit ,unbewußter' Verkündigung gemeint ist, soll ein Zitat aus einem Hörer-Brief an die Abteilung Religion/Hörfunk des ORF im Anschluß an eine ,Menschenbilder'-Sendung verdeutlichen:

"Kein einziges Mal wurde von dieser einfachen Frau aus dem Volk ein religiöser Begriff verwendet - aber durch ihre Lebensgestaltung und ihre Art, wie sie darüber erzählt, wird für mich im Erinnern des Gehörten eine starke religiöse Kraft fühlbar [...] - jedenfalls hat ihre Darstellung mich unmittelbar im Herz berührt."4

Verkündigung geschieht darüber hinaus vor allem in der Predigt, der Katechese, im liturgisch-sakramentalen Zeichen, im seelsorglichen Gespräch und im einfachen spontanen Zeugnis in Wort und Tat.

Seit den Anfängen des Christentums bedient sich die Verkündigung des jeweiligen Kommunikationsraumes und hat auch die jeweils gegebenen Mittel zu nutzen verstanden: vom Sendschreiben des Paulus über den Buchdruck bis hin zu den audiovisuellen Medien wie Radio/Fernsehen. 5

Um die Möglichkeiten der Verkündigung via Radio klar zu

3 Vgl. Th. Filthaut, Verkündigung - Theologisch, in: Handbuch theologischer Grundbegriffe, Bd. II, München 1963, 763-768.

4 Zit.n. H. Gaisbauer, Seelsorge durch das Radio?, Vortragsmanuskript (im Besitz der Autorin), 12.

5 Vgl. dazu W. Petkewitz, Verkündigung in der Mediengesellschaft. Neue Informations- und Kommunikationstechniken in der kirchlichen Praxis, Gütersloh 1991, 229: „Verkündigung, die 'die Situation des Hörers real verändert', die zumindest von ihrer Kommunikationsstruktur her in der Lage ist, kann unter den Bedingungen einer 'Informationsgesellschaft' nur personale Verkündigung sein. Der Umgang mit neuen Informations- und Kommunikationstechniken sollte davon als Form der Öffentlichkeitsarbeit unterschieden und durch klare medienpolitische Konzepte geregelt werden." (Hervorhebungen von mir) 
definieren, ist es sinnvoll, mit Petkewitz das Verkündigungsgeschehen von einem kommunikationstheoretischen Standpunkt her zu beleuchten. Petkewitz teilt das Verkündigungsgeschehen in Anrede (z.B. Predigt), Darstellung (z.B. Gottesdienst), Dialog (z.B. Seelsorge) und Sprache des Tuns (Diakonie) ein. ${ }^{6}$

Verkündigung durch das Radio hat einen grundsätzlich monologischen Charakter, welcher durch die Eigenart des Mediums hervorgerufen ist (räumliche Trennung; kein direkter Kontakt zwischen Gestaltenden und Hörenden, ausgenommen bei Telefonkontakt). Radioverkündigung ist daher primär Verkündigung als Anrede.

Ziel der Radioverkündigung muß es daher sein, einen inneren Dialog' mit den HörerInnen aufzubauen - d.h. mit den Grundgefühlen der Menschen zu kommunizieren, oder, mit dem Dichter Paul Celan gesprochen, $\mathrm{zu}$ versuchen, "Herzland ${ }^{\prime 7} \mathrm{zu}$ erreichen. Erst dadurch bekommt die Radioverkündigung auch einen darstellenden, dialogischen und wirklichkeitsverändernden Charakter. (Erst dann lebt z.B. die Auslegung des Sonntagsevangeliums in der Sendung ,Erfüllte Zeit'.)

Um in diesen inneren Dialog eintreten zu können, sind mehrere Komponenten im Verkündigungsgeschehen mitbestimmend. Sie werden im folgenden besprochen:

- Hilfreiche und hemmende Wirkung des Mediums Radio auf die Verkündigung

- Glaubwürdige und authentische Sprache

- Zeitgemäße Verkündigungsinhalte

\section{Hilfreiche und hemmende Wirkung des Mediums Radio auf die Verkündigung}

\subsection{Ansichten in Form bringen und hörbar machen - Parallelen zwischen Verkündigung und Medium Radio}

Eine ,Diagonal'-Sendung von ,Österreich 1 ' beschäftigte sich einmal mit dem Thema ,Radio-Hören':

"Radiohören läuft entlang einer Zeitschiene. Nach 7 kommt 8, man kann das nicht umdrehen, man kann beim Radiohören nicht zurückblättern. Es ist ein klassisch analoges Medium. Hören heißt notgedrungen, sich einem Weg anzuvertrauen, den andere gewählt

6 Ebd., 43-63.

7 P. Celan, Ansprache anläßlich der Entgegennahme des Literaturpreises der Freien Hansestadt Bremen, in: B. Allemann/S. Reichert (Hg.), Paul Celan. Gesammelte Werke in fünf Bänden, Bd. 3, Frankfurt/M. 1983, 185. 
haben. [...] Die menschliche Rede ist geprägt von Umwegen, man hastet vor, bleibt hängen, sucht Worte, wiederholt sich, macht Gedankensprünge. Nirgends kann man das grundsätzlich so pur erleben wie im Radio. Man kann im Radio frei sprudelnde Geschichten hören, die ganz anders klingen würden, würde man sie aufschreiben und dann lesen oder vorlesen. [...] Radio hören heißt: anderen beim Denken zuhören. ${ }^{\lrcorner 8}$

Bei der Verkündigung geht es ebenfalls darum: hinzuhören, mitzudenken, sich einem vorerzählten Weg anzuvertrauen. Verkündigung kommt zuerst vom Hören und richtet sich dann an ein ,offenes Ohr'. Verkündigung lebt auch von der gesprochenen Rede, vom Erzählen, Schildern und Erinnern. Dafür ist das ,analoge Wesen' des Radios hilfreich.

Das Radio ist abhängig vom Ein- und Ausschalten, vom Wollen der Hörenden. Ebenso ist das Verkündigungsgeschehen abhängig von der Bereitschaft der Hörenden zuzuhören.

Über das Radio werden tausende von HörerInnen erreicht, der Moderator oder die Musik wenden sich jedoch tausendmal an jede/ $\mathrm{n}$ einzelne/n. Verkündigung richtet sich ebenfalls grundsätzlich an alle Personen, kommuniziert aber im Grunde mit jeder/jedem einzelnen.

Radio vermittelt das Gefühl: Ich bin nicht allein (im Englischen deutlicher: "alone together"). Es begleitet den Menschen im Alltag sowie bei alltäglichen Lebensvollzügen. Dazu ein Beispiel:

"Eine menschenleere Wohnung. Eine junge Frau betritt den Raum, dreht das Licht an und sogleich auch den portablen Rundfunkempfänger, den sie, als sie morgens das Haus verließ, am Tisch neben dem Telefon abgestellt hatte. Dann erst legt sie Mantel und Schuhe ab, geht in die Küche, um Kaffee aufzustellen. Licht und Musik sind im Raum, und zwischendurch Stimmen. Stimmen aus dem kleinen Portablen ..., sie zu hören ist wichtig, sie zu verstehen zweitrangig. Musik und Stimmen machen, daß Angst und Stille schweigen; im Gefühl: ich bin da und du bist da und nicht allein. So äfft der kleine Portable Gott nach: ,Ich bin das ich bin da!' 9

Das Radio kann demnach als "elektronisches Amulett"10 bezeichnet werden, aber auch als Medium mit einer seelsorglichen' Dimension. Wenn das Anliegen christlicher Seelsorge das Dasein und Begleiten im

8 "Radio hören heißt: anderen beim Denken zuhören.", „Diagonal’ v. 28.5.1994, Ö1.

9 H. Gaisbauer, Seelsorge durchs Radio?, in: Theologische Information (Beilage zum Linzer Diözesanblatt), Diözese Linz - Institut für Pastorale Fortbildung (Hg.), Linz 1993, 4.

$10 \mathrm{Vgl}$. H. Albrecht, Die Religion der Massenmedien, Stuttgart/Berlin/Köln 1993, 63. 
Leben ist, so kann das Radio darum christliche Seelsorge unterstützen (z.B. regelmäßige Gottesdienstübertragungen in das Zimmer einer alten Person, oder Sendungen von persönlichen Glaubens- und Lebenszeugnissen in die Wohnung von einsamen Personen).

Für die Vermittlung der Verkündigungsinhalte können neben dem Wesen des Mediums auch bestimmte radiojournalistische Formen unterstützend wirken. Das Feature z.B. versucht, "Ansichten von dieser Welt in Form zu bringen"11 und hörbar zu machen. Für die Umsetzung stehen dabei alle im Hörfunk möglichen stilistischen Mittel zur Verfügung. Diese Form des Features wird vor allem der Komplexität von religiösen Themen gerecht.

Wenn Verkündigung die Kooperation mit dem Medium eingeht und dessen Unter-stützung möchte, dann erfordert dies auch Kenntnis und Gespür für die Eigenheiten des Mediums.

\section{2 "Das Evangelium läßt sich nicht in eine Show pressen"12 - Verkündigung zwischen Moderation, Musik und Werbung}

Der Vorwurf, daß bestimmte Programmformate und Programmschemata religiöse Sendungen und vor allem deren Inhalte verzerren oder sogar verstümmeln, richtet sich vor allem gegen jene Sender, in denen religiöse Kurzbeiträge von zwei bis drei Minuten ohne feste Sendeplätze zwischen Musik, Werbung und Moderation eingestreut werden.

Durch Zielsetzungen dieser Sender können viele religiöse Themen und Inhalte dort nicht angesprochen werden. Bei manchen Themen würde eine längere Sendezeit und weniger ,Hektik' im Umfeld der Sendung benötigt werden. In zweieinhalb Minuten ist aber dennoch Verkündigung möglich, die vor allem pointierte christliche Antwortmodelle auf Lebensfragen vorstellen kann oder helfen kann, „für einen kurzen Moment inne zu halten".13

Eine religiöse Sendung muß sich darüber hinaus als solche auch zu erkennen geben. Bieger stellt dazu fest, daß grundsätzlich jede Sendeform die Möglichkeit hat in sich religiös zu sein:

11 Chr. Hülsebus-Wagner, Feature und Radio-Essays. Hörfunkformen von Autoren der Gruppe 47 und ihres Umkreises (Cobra-Medien 1), Aachen $1983,49$.

12 G. Biemer, Verkündigung ohne Begegnung? Zum Verhältnis von Kirche und Medien, in: Diakonie 17 (1986) 365.

13 B. Tups, Kirche im Privatfunk kommt an. Kirchliche Sendungen in niedersächsischen Privatsendern, in: Communicatio Socialis 25 (1992) 400. Vgl. auch: U. Harprath, Zwischen Einschaltquoten und kirchlichem Auftrag. Das Engagement der bayrischen Diözese im privaten Hörfunk, in: Communicatio Socialis 24 (1992) 184-192. 
"Als religiöse Rede kann Rede erst dann unterschieden werden, wenn sie den religiösen Bezug möglichst im Rückgriff auf die Situation, in der dieser Bezug erkannt, erfahren, übernommen wurde, zum Ausdruck bringt - oder wenn über diese Situation berichtet wird."14

Es ist also nicht nur eine bestimmte journalistische Form geeignet, religiöse Inhalte zu präsentieren.

Es erfordert Kreativität, Können und auch etwas Glück, um in zweieinhalb Minunten religiöse Inhalte unverzerrt und zugleich spannend sowie unterhaltsam (so daß sie auch in einem Unterhaltungsprogramm gehört werden) darzustellen.

Für den praktischen Theologen Manfred Josuttis entscheidet sich an der Verkündigungssprache, ob eine Sendung unterhaltsam ist oder nicht. Verkündigungssprache ist zwar keine Showsprache, aber sie kann sehr wohl unterhaltsam sein. Das bedeutet für ihn: voll (Lebens-)Kraft und (Lebens-)Mut. ${ }^{15}$ Josuttis weist darauf hin, daß die Gleichnisse Jesu oder die Erlebnisse der Jüngerinnen und Jünger mit Jesus auch von zwei bis drei Minuten Dauer waren. Er betont, daß die Situation aus der persönlichen Begegnung wuchs. „Die Pointen, die Zuspitzungen, die bedeutungsvollen Geschichten schaffen [aber] - und das kann man gut innerlich nachkonstruieren - einen erfüllten Augenblick." ${ }^{16} \mathrm{Um}$ diesen erfüllten Augenblick via Radio ankommen zu lassen, bedarf es der Ohr-Kontakte mit den Hörenden und solcher Sätze, die im Vertrauen auf ihre Kraft hinausgesagt werden. Solche Sätze müssen spannungsreiche und unterscheidende Sätze sein. Sie brauchen vor der Ambivalenz der Realität nicht zurückzuschrecken, sondern sollten sie herausstreichen. Es sind Sätze, die ein klares Ja und Nein sagen. Inhaltlich ist ein klares Maß an Offenheit, aber auch die Fähigkeit zur Abgrenzung gefordert:

„Von Gott reden vor dem Eschaton, vor dem Einbruch des Reiches Gottes, heißt immer auch und unbedingt nicht etwas Grenzenloses, Rieselndes, Fließendes, Rauschendes (das ist im Eschaton so, die Engel erleben das vielleicht so), sondern etwas Kantiges, Eckiges, Sperriges. Der Einbruch Gottes unterbricht Alltagsabläufe, stört in der Routine und hilft gerade deshalb zum Leben."17

14 E. Bieger, Religiöse Rede im Fernsehen (Anm. 2), 372.

$15 \mathrm{Vgl}$. M. Josuttis, Unterhaltsam von Gott reden, in: ORF Abteilung Religion (Hg.), Von Gott im Radio und Fernsehen reden, Graz/Budapest 1992, $32-45$.

16 Ebd., 40.

17 Ebd., 37. 


\section{Glaubwürdige und authentische Verkündigung - Die Sprache}

Um die Inhalte unverfälscht bei den Hörenden ankommen zu lassen und um einen inneren Dialog aufzubauen, benötigt die Verkündigung ein glaubwürdiges und authentisches Sprechen.

Die glaubwürdige und authentische Präsentation via Medium wird von vielen Menschen oft als das alleinige Rezept gegen das schwindende Kircheninteresse angeführt. Mit ,glaubwürdig' wird im allgemeinen ein aufrichtiges, ehrliches und packendes Erzählen vom eigenen Glaubensleben verbunden. Dazu gehört aber auch (vor allem in Bezug auf das Massenmedium Radio), daß diejenige Person einen guten Tag hat, keine Angst vor dem Mikrophon, von sich erzählen kann, eine sprudelnde und packende Redeweise hat, und daß der/die Hörende genau das Verkündete hören will. Viele glaubwürdige Personen kommen dadurch oft nicht so ,glaubwürdig' und von den Hörenden als authentisch erlebt durch das Radio an.

Die Arbeit an einer glaubwürdigen und authentischen Verkündigung beginnt beim Sprechen jeder verkündenden Person. Verkündigung ist dann authentisch, wenn der eigene Gedankengang sprachlich transparent gemacht wird. Alois Vergeiner von der ORF Abteilung Religion erklärt dies seinen Sprecherinnen und Sprechern folgendermaßen:

„Der Dialog muß sich zuerst in mir abspielen, das heißt: ich muß zuerst mit mir selber reden, ich muß den Denkprozeß in mir selber durchmachen. Spannender und verbindlicher, weil offener ist es, wenn ich den anderen in den Denkprozeß hineinschauen lasse, der in mir vorgegangen ist, den anderen in den Denkrythmus einbeziehe." 18

Die Folge davon ist eine dialogische Sprache, die einer klischeehaften oder triumphalistischen Sprache widerspricht. Sie fordert die Sprechenden heraus und stellt hohe Anforderungen an sie, denn es ist z.B. leichter, Vorgeformtes zu wiederholen, als eigene Denkprozesse transparent $\mathrm{zu}$ machen. Es kann aber nur so eine Vertrauensbasis zu den HörerInnen aufgebaut werden.

Die Verkündigung kommt auch aus diesem Grund nicht ohne das Genre des Erzählens aus, denn im Erzählen liegt die Möglichkeit, mehr als reine Worte zu Gehör zu bringen. „Das Herz wird gewöhnlich erreicht nicht durch Vernunft, sondern durch Imaginationskraft, durch Zeugnis, Geschichte, Beschreibung; Personen überzeugen uns, Stimmen tauen uns auf."19

18 Zit.n. einem von der Autorin geführten Interview mit Alois Vergeiner (Mitarbeiter der ORF-Abteilung Religion/Hörfunk) v. 01.02.1995.

19 J. H. Newman, zit.n.: H. Gaisbauer, Von Gott reden in Radio und Fernsehen, ORF-Abteilung Religion (Hg.), Graz/Budapest 1992, 12. 
Stimmen können auch ohne direkte Begegnung mit den sprechenden Personen über das Radiogerät auftauen. Ein so geartetes glaubwürdiges und authentisches Sprechen ermöglicht es, die personale Mysteriendimension und innerste Erlebnisse hörbar werden zu lassen. ${ }^{20}$

Es darf dabei nicht die Rolle des/der Sendungsgestalters/in übersehen werden. Sie bewegt sich zwischen Management und Verkündigung. Die Entscheidungskompetenzen liegen zum Großteil bei ihm/ ihr. Ihre Aufgabe ist die Koordination zwischen Technik, Thema und Sprechenden, sowie deren Auswahl. Sie leisten Dienst an der Verkündigung, können aber als Glaubende und „Mitwandernde des Gottesvolkes $^{\prime \prime 21}$ auch Verkündende sein, wenn sie ihren Glauben in Zwischentexten, Musik sowie Gestaltung transparent machen. Sie sind darüber hinaus die ersten Zuhörenden, die oft dazu beitragen, daß die verkündenden Stimmen wirklich authentisch ankommen, indem sie z.B. einfach nur im unpersönlichen Aufnahmestudio neben den Sprechenden sitzen und zuhören. Auch das vermittelt das Gefühl, nicht zur Maschine, sondern zu einem Menschen zu sprechen.

\section{Zeitgemäße Verkündigungsinhalte}

Eine Verkündigung, die nicht bei den gegenwärtigen Fragen, Positionen, Stimmungen und Sehnsüchten der Menschen ansetzt, wird diese Menschen auch schwer erreichen können. Danach hat sich auch der Verkündigungsinhalt auszurichten. Religiöse Aussagen bewegen sich grundsätzlich zwischen Welthaftem und Nichtwelthaftem, zwischen Dauerhaftem und Zeitgemäßem.22 Verkündigung durch das Massenmedium Radio ist auch vor die Frage gestellt: Wie sag ich die gleichbleibende Botschaft, so daß sie auch heute verstanden wird? Was sind die gegenwärtigen Fragen und die christlichen Antworten?

Vordergründig herrscht in der gegenwärtigen Gesellschaft Zufriedenheit über die Möglichkeit, z.B. mit modernen Kommunikationsmitteln eigene Grenzen zu überwinden, vom eigenen Wohnzimmer aus in die ganze Welt zu schauen, Grenzen nicht beachten zu müssen und das Leben zu genießen.

Im Inneren der Menschen ist oft eine pessimistische Grundstimmung bemerkbar, die von Angst, Ohnmachtsgefühlen und Heimatlosigkeit begleitet ist.

20 Dies behaupte ich auch gegen die Auffassung G. Biemers in dem bereits zitierten Aufsatz Verkündigung ohne Begegnung? Zum Verhältnis von Kirche und Medien, in: Diakonie 17 (1987) 362-367.

21 Aus einem von der Autorin geführten Interview mit $\mathrm{H}$. Gaisbauer (Leiter der ORF-Abteilung Religion/Hörfunk) v. 11.02.1995.

22 Vgl. R. Guardini, Die religiöse Sprache, in: M. Kaempfert (Hg.), Probleme der religiösen Sprache (Wege der Forschung 442), Darmstadt 1983, 50-71. 
Der Mensch übt sich gegenwärtig in der Kunst: „zufrieden mit der Resignation, Angst und Zukunftssorge umzugehen. Cool leben, ohne dabei $\mathrm{zu}$ frieren“23, so formuliert es der Soziologe Bernd Guggenberger.

Dies äußert sich auch in der Einstellung der Religion gegenüber und im religiösen Engagement. Religion wird zur Privatsache und hat zunehmend fundamentalistische oder pantheistische Züge. Das gemeinsame Ziel dieses Mischglaubens ist es erlöst zu werden, denn ,Engagement nützt nichts mehr'. Die Bedeutung der "organisierten Religion" (wie der christlichen Kirchen) nimmt ab und wird an die „Randzonen der Gesellschaft“24 gedrängt. Zum Teil übernehmen die Massenmedien die Funktion einer Religion. „Hier wird aus der Enge realer Alltäglichkeit und Entsinnlichung das Gefühl der Weite und Autonomie, das Gefühl omnipräsenter Teilnahme geweckt.“25 Es wird das ,ganz Andere' garantiert, das der Mensch ,braucht'. Dies gilt zum Teil auch für die modernen Kommunikationsmittel (Computer, Computervernetzung, Computersimulationen).

Die Kirchenkritik vieler Zeitgenossen zeigt eine Antipathie gegenüber Bevormundung aber andererseits auch eine Barriere des Menschen: "die Weigerung des Denkens, bis an die ihm gezogene Grenze $\mathrm{zu}$ gehen und in die es dort erwartende Transformation einzuwilligen."26 Das wird für Eugen Biser auch am Verstummen des Atheismus als Gegenstück des Glaubens deutlich. Der wahre Gegensatz zum Glauben ist heute nicht der Atheismus, sondern die Angst, die fehlende Lebenskraft und die Gleichgültigkeit.

Christliche Medienverkündigung hat daher drei Aufgaben:

a) Die Sache, das spezifisch Christliche noch immer und wieder zu verkünden. Kardinal Daneels fordert auf: „Wir sollen schlicht und einfach bezeugen, was wir sind."27 Das ist die Aufforderung zum Zeugnis des Glaubens an den menschgewordenen Gott in Jesus Christus, den Glauben an ein personales $D u$, den Glauben an die persönliche Grenze, an die Endlichkeit des Lebens, die durch die Auferstehung nach dem Tod gesprengt wird.

23 B. Guggenberger, Sein oder Design. Zur Dialektik der Abklärung, Berlin 1987, 29.

24 Vgl. E. Biser, Glaubensprognose. Orientierung in postsäkularisierter Zeit, Graz/Wien/Köln 1991, 43.

25 W. Schmidt, Opium des Volkes? Über die Medienreligion und ihre strukturelle Entzauberung des Alltags, in: N. Janowski (Hg.), Die kanalisierte Botschaft. Religion in den Medien - Medienreligion, Gütersloh 1987, 106. E. Biser, Glaubensprognose (Anm. 24), 123.

27 G. Danneels, "Wir sollen schlicht und einfach bezeugen, was wir sind.", in: Herder-Korrespondenz 48 (1994) 133-138. 
b) Das Zeitgemäße dieser Botschaft erzählen: die heilende, angstüberwindende, hoffnungsspendende Kraft des Glaubens an Gott.

c) In einer Sprache verkünden, die durch ein Massenmedium gehört wird, weil sie persönlich, transparent und spannungsreich ist.

In einer Analyse28 religiöser Sendungen des ORF Hörfunk wurde gefragt, inwieweit in diesen Sendungen ein umfassender Glaube (a. und b.) vermittelt wird. In den meisten Sendungen wurde ein umfassender Glaube verkündet; es wurde aber deutlich, wie eng Inhalt und Form (damit ist Sprache und übrige Gestaltung gemeint, vgl. c.) verbunden sind.

\section{Schlußbemerkung}

Das Radio ist ein geeignetes Medium für die christliche Verkündigung. Durch das Radio und andere Massenmedien geschieht eine zeitgemäße Verkündigung in den Alltag der Menschen und nicht in einen sakralen Raum hinein.

Verkündigung durch ein Massenmedium wäre aber überfordert, wenn sie der einzige Kontakt zur Gesellschaft wäre. Kirche ohne Gemeindeleben, ohne Gemeinschaft ist leer. Deshalb kann Verkündigung durch die Medien nur kirchlich sein, weil sie von der Gemeinschaft der Glaubenden lebt. Meiner Meinung nach ist es daher falsch zu glauben, glaubwürdige Kirchenpräsenz in den Medien sei das Allheilmittel gegen mangelndes Kircheninteresse der gegenwärtigen Menschen.

Verkündigung durch das Radio wird sich mit dem Medium Radio verändern. Auf der Berliner Funkausstellung wurde bereits das ,digitale Radio' vorgestellt. Durch die digitalen Übertragungsmöglichkeiten werden „zusätzliche Übertragungsmöglichkeiten für Texte und sogar Bilder, Daten und graphische Darstellungen im Radio (eröffnet). Allerdings ist für den Empfang eine neue Gerätegeneration erforderlich." 29

Die mediale Veränderung hat Auswirkungen auf die Sprachwahl der Verkündigung und auf die Verkündigungsinhalte. Christliche Weltanschauung steht zunehmend neben anderen Deutungs- und Gestaltungsmustern des Lebens, eben auch neben der ,Weltsicht ' durch die modernen Kommunikationsmittel. Daher muß sich die christliche Verkündigung auch die Frage stellen lassen:

„Mit welchem Recht kann die christliche Verkündigung mehr

28 Vgl. G. Cakl, Verkündigung im Alltag (Anm.1), 73-90.

29 Zit. n. P. A. Wallnöfer, in: Salzburger Nachrichten, 50 (02.09.1995) III. 
Glaubwürdigkeit beanspruchen als die,präsentische Eschatologie‘ [das Reich Gottes auf Erden] komplexer Rollenspiele [z.B. Computerspiele/ Virtual Reality] in denen man auf Knopfdruck aufstehen kann, wenn das ,Leben' in der Simulation zu Ende ist?" 30

\section{SUMMARY: Proclamation of faith by radio}

Radio and Christian preaching of the Good News aim at the ear of men and women. Alone by reason of common hearing, there is a cooperation. In this article, present Christian preaching and its presence in media is reflected. The reflections are based on a clear definition of Christian preaching. As consequence of the monoloque-structure of radio, radio-preaching can only be of addressing character to the listeners. Preaching, however, is aiming at an ,inner dialogue' with the listeners. In this context, several components are listed: 1 . helpful or hampering influences of the medium on preaching; 2 credible language which makes faith transparant; 3 . up-to-date contents of the Good News.

Via radio spreading of the Good News in todays' language for everydays' life is possible. To expect full proclamation of faith via radio as the single contact to the Church, would be overtaxing radio.

\section{RÉESUMÉ: Proclamation à la radio}

La radio et la proclamation chrétienne dirigent leurs messages vers les oreilles de l'homme. Cette raison audible fait qu'ils coopèrent déja ensemble. L'article thématise la proclamation chrétienne actuelle et sa présence dans les médias. On part ici d'une définition claire de la proclamation. De par la structure monologique de la radio, la proclamation à la radio ne peut avoir qu'um caractère d'approche. Mais le but de cette proclamation doit être d'avoir un "dialogue interne ${ }^{\prime}$ avec les auditeurs. On cite plusieurs composantes: 1 . effet serviable et gênant du média; 2 . authentique, un langage qui rend les idées transparentes; 3 . contenus actuels de la proclamation.

Par la radio, les proclamations modernes s'introduisent dans la vie quotidienne des hommes. On en demanderait trop à la proclamation, si elle devait être le seul contact de l'eglise avec la société.

\section{RESUMEN: La predicación por la radio}

La radio y la predicación cristiana se dirigen al órgano de la audición. Ya sólo por esta razón auditiva ambas entran en cooperación. El escrito tematiza la predicación cristiana actual y su presencia en los medios. En esto se parte de una clara definición de la predicación. Debido a la estructura monológíca de la radio, las prédicas por la radio sólo pueden tener caracter de alocución. Pero el objetivo de estas prédicas tiene que ser el entablar con el auditor un "diálogo interno". Se nombran varios componentes: $1^{\circ}$ la ayuda y el efecto inhibidor del medio para la prédica; $2^{\circ}$ un idioma digno de credito que haga transparente el pensamiento; 3 un contenido adecuado al tiempo que se vive.

A través de la radio la predicación penetra en la cotidianeidad des los hombres. Pero la predicación por la radio se disvirtuaría si se convietiera en el único contacto de la iglesia con la sociedad.

30 Chr. Wessely, Virtual Reality und christliche Theologie - Theotechnologie, in: Theologisch-praktische Quartalsschrift 143 (1995) 243. 